\title{
Could mean platelet volume be used as a marker for activity and severity index of alopecia areata?
}

\author{
Nebahat Demet Akpolat ${ }^{\oplus}$ \\ Dermatology, Beykoz State Hospital, İstanbul, Turkey.
}

\begin{abstract}
Background. We aimed to determine whether MPV can be used as a marker for the activity and the severity index of alopesi areata (AA).

Method. The charts of 71 children who received a diagnosis of AA and 70 age and gender-matched healthy children were retrospectively evaluated. The severity of hair loss was classified as S1 $(<25 \%), \mathrm{S} 2(25-49 \%), \mathrm{S} 3$ (50-74\%), S4 (75-95\%), S4b (96-99\%) (according to the percent of the area involved), alopecia totalis (AT), and alopecia universalis (AU). In the laboratory tests, the results of the complete blood count, anti-nuclear antibody (ANA), thyroid function tests (TSH, free/total T4, free/total T3), and autoimmune thyroid antibodies [antithyroid peroxidase antibody (anti-TPO) and anti-thyroglobulin antibody (AT)] were recorded.
\end{abstract}

Results. A total of 141 cases including 61 (43.3\%) males and 80 (56.7\%) females were included. There was no statistically significant difference between the groups according to the mean age ( $p>0.05)$.

The MPV measurements were statistically significantly higher in the AA group $(p<0.01)$. There was no statistically significant difference between the types of AA according to the mean age, gender distribution, the presence of nail involvement, the presence of family history, and the presence of autoimmune disease $(\mathrm{p}>0.05)$. There was no statistically significant difference between the severity of AA according to the mean age, gender distribution, the presence of nail involvement, the presence of family history, and the presence of autoimmune disease $(\mathrm{p}>0.05)$.

Conclusion. MPV is helpful in assessing clinical activity in patients with AA. However, prospective studies involving more patients are needed to support our findings.

Key words: alopecia areata, mean platelet volume, inflammation.

\begin{abstract}
Alopecia areata (AA) is a chronic autoinflammatory disease that is characterized by episodic and non-scarring loss of the scalp, eyebrows, eyelashes and other body hairs and by sharply demarcated patches. ${ }^{1}$ Although its etiopathogenesis remains unclear, the autoimmune reactions, which target the hair matrix, are thought to be responsible for genetically susceptible individuals. ${ }^{2}$ The lifetime risk of $\mathrm{AA}$ is $1.7-2 \%$, regardless of age and sex. ${ }^{3,4} \mathrm{AA}$ is seen especially in young
\end{abstract}

Nebahat Demet Akpolat
drdemetakpolat@gmail.com

Received 2nd May 2017, revised 23th April 2017, accepted 1st May 2017. patients and approximately $60 \%$ of patients have their first attack before the age of $20.5 \mathrm{AA}$ can also be accompanied by other autoimmune diseases such as autoimmune thyroid diseases, vitiligo, and lupus erythematosus. ${ }^{6}$ Mean platelet volume (MPV) is used as part of the complete blood count in full blood count analyzers and is a common marker used to demonstrate platelet function and activation. ${ }^{7}$ It has been shown in recent years that MPV is high in chronic inflammatory diseases and may be used as an inflammatory marker. ${ }^{8}$ In this study, we examined the relationship between MPV level and disease severity, nail involvement, family history, disease duration and other accompanying autoimmune diseases in pediatric patients with AA. 


\section{Material and Methods}

After receiving the ethical board approval (no:10, in 19.10.2015) of Istanbul Anatolia-North Region Public Hospitals Trust, 71 children under the age of 18 who received a diagnosis of AA at the Dermatology outpatient clinic of Beykoz State Hospital between 2015 and 2017 were retrospectively evaluated in terms of age, sex, disease severity, lesion distribution, nail findings, family history, and accompanying autoimmune diseases. The control group included 70 age and gender-matched healthy children who had no dermatologic or systemic disease. If the duration of AA was 3 months or less, it was considered acute. If the duration of AA was over 3 months, it was considered chronic. The severity of hair loss was classified as S1 $(<25 \%)$, S2 (25-49\%), S3 (50-74\%), S4 (75$95 \%), S 4 b(96-99 \%)$ (according to the percent of the area involved), alopecia totalis (AT), and alopecia universalis (AU). In the laboratory tests, the results of the complete blood count, anti-nuclear antibody (ANA), thyroid function tests (TSH, free/total T4, free/total T3), and autoimmune thyroid antibodies [anti-thyroid peroxidase antibody (anti-TPO) and antithyroglobulin antibody (AT)] were recorded.

\section{Statistical Analysis}

NCSS-2007 software program (Number Cruncher Statistical System) (Kaysville, Utah, USA) was used for statistical analysis. Mean, standard deviation, median, frequency, ratio, minimum, and maximum were used for comparing the descriptive data. Moreover, the Mann Whitney U test was used for comparing the parameters without normal distribution between two groups. The Kruskal Wallis test was used for comparing three or more groups without normal distribution. The FisherFreeman-Halton test, Fisher's Exact test, and Perason chi-square test were used for comparing the qualitative data. The significance level was considered as $\mathrm{p}<0.05$.

\section{Results}

A total of 141 cases including $43.3 \%(n=61)$ males and $56.7 \%(n=80)$ females who were admitted to the Dermatology outpatient clinic of Beykoz State Hospital between 2015 and 2017 were enrolled in the study. Their ages ranged from 1 to 18 years and the mean age was $12.57 \pm 4.38$ years.

In the AA group, their ages ranged from 1 to 18 years and the mean age was $12.24 \pm 4.69$ years. In the control group, their ages ranged from 2 to 18 years and the mean age was $12.91 \pm 4.05$ years. There was no statistically significant difference between the groups according to the mean age ( $>0.05)$ (Table I).

In the AA group, the MPV measurements ranged from 6.4 to 11.4 and the mean MPV level was $8.33 \pm 1.15$. In the control group, the MPV measurements ranged from 5.4 to 7.7 and the mean MPV level was $6.33 \pm 0.41$. The MPV measurements were statistically significantly higher in the AA group ( $\mathrm{p}=0.001 ; \mathrm{p}<0.01)$ (Table I), (Fig. 1).

The MPV measurements showed no statistically significant differences according to the type of AA ( $p=0.344 ; p>0.05)$ (Table II). While the MPV measurements showed no statistically

Table I. Age and MPV evaluations according to groups.

\begin{tabular}{llccc}
\hline & & \multicolumn{2}{c}{ Group } & \\
\cline { 3 - 4 } Age & Min-Max (Median) & Alopeci areata $(\mathrm{n}=71)$ & Control $(\mathrm{n}=70)$ & \multirow{2}{*}{0.551} \\
& Mean \pm SD & $1-18(13)$ & $2-18(14)$ & \\
\multirow{2}{*}{ MPV } & Min-Max (Median) & $12.24 \pm 4.69$ & $12.91 \pm 4.05$ & \multirow{2}{*}{$0.001^{* *}$} \\
& Mean \pm SD & $6.4-11.4(7.9)$ & $5.4-7.7(6.4)$ & $6.33 \pm 0.41$ \\
\hline
\end{tabular}

${ }^{a}$ Mann Whitney U Test, ${ }^{* *} \mathrm{p}<0,01$

MPV: Mean platelet volume 


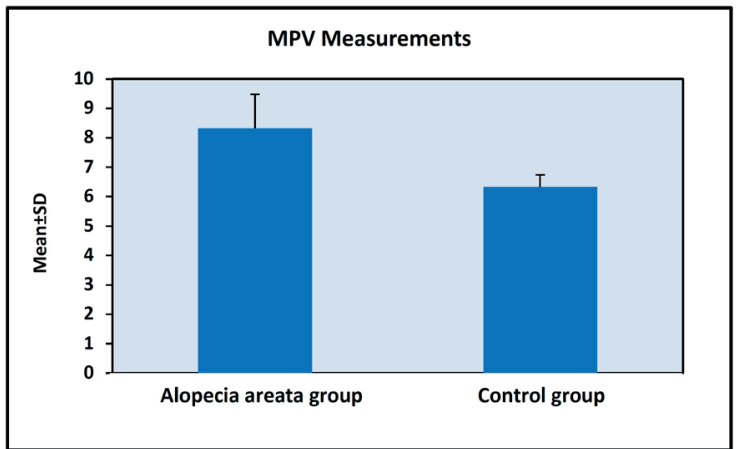

Fig. 1. Distribution of MPV measurements according to groups.

significant difference according to the severity of AA ( $>0.05)$ (Table II), it was remarkable that the MPV measurements were high in the patients with AA severity S2.

The MPV measurements showed no statistically significant difference according to the presence of nail involvement, the presence of family history, the presence of autoimmune disease, and disease duration $(\mathrm{p}>0.05)$ (Table II).

There was no statistically significant difference between the types of AA according to the mean age and gender distribution $(p>0.05)$ (Table III).
There was no statistically significant difference between the types of AA according to the presence of nail involvement, the presence of family history, and the presence of autoimmune disease $(p>0.05)$ (Table III). It was remarkable that the patients who had a reticular type of AA had a higher presence of nail involvement than patients who had a siasphio or a ophiasic type of AA (Table III).

There was no statistically significant difference between the severity of AA according to the mean age and gender distribution $(p>0.05)$ (Table IV).

There was no statistically significant difference between the severity of AA according to the presence of nail involvement, the presence of family history, and the presence of autoimmune disease $(p>0.05)$ (Table IV).

\section{Discussion}

AA is a chronic inflammatory disease that is characterized by episodic and non-scarring loss of the scalp and/or other hairy areas of the body. ${ }^{9}$ Although genetic predisposition, non-specific immune reactions, organ-specific autoimmune reactions, environmental factors, and atopy

Table II. Evaluations of descriptive characteristics according to MPV measurements.

\begin{tabular}{|c|c|c|c|c|}
\hline & & \multicolumn{2}{|c|}{ MPV } & \multirow{2}{*}{$\mathrm{p}$} \\
\hline & & Min-Max (Median) & Mean \pm SD & \\
\hline \multirow{3}{*}{ Type of alopecia areata } & Reticular & $6.4-11.4(8.4)$ & $8.57 \pm 1.25$ & \multirow{3}{*}{ b0.344 } \\
\hline & Ophiasis & $6.8-10.2(7.7)$ & $8.26 \pm 1.16$ & \\
\hline & Siasphio & $6.8-10.5(7.7)$ & $8.07 \pm 0.99$ & \\
\hline \multirow{2}{*}{ Severity of alopecia areata } & S1 & $6.8-10.5(7.7)$ & $8.05 \pm 1.10$ & \multirow{2}{*}{ a 0.085} \\
\hline & S2 & $6.4-11.4(8.5)$ & $8.52 \pm 1.16$ & \\
\hline \multirow{2}{*}{ Nail involvement } & No & $6.4-11.4(7.9)$ & $8.39 \pm 1.19$ & \multirow{2}{*}{ a 0.522} \\
\hline & Yes & $6.8-9.9(8.2)$ & $8.12 \pm 1.03$ & \\
\hline \multirow{2}{*}{ Family history } & No & $6.4-11.4(7.9)$ & $8.36 \pm 1.19$ & \multirow{2}{*}{ a 0.642} \\
\hline & Yes & 7.2-9.4 (8) & $8.06 \pm 0.76$ & \\
\hline \multirow{2}{*}{ Autoimmune disease } & No & $6.4-11.4(8)$ & $8.30 \pm 1.13$ & \multirow{2}{*}{ a 0.655} \\
\hline & Yes & $6.8-10.9(7.9)$ & $8.49 \pm 1.29$ & \\
\hline \multirow{2}{*}{ Disease duration } & Acute hair loss & $6.8-10.5(8.5)$ & $8.47 \pm 1.07$ & \multirow{2}{*}{${ }^{\mathrm{a}} 0.166$} \\
\hline & Chronic hair loss & $6.4-11.4(7.7)$ & $8.16 \pm 1.25$ & \\
\hline
\end{tabular}

${ }^{a}$ Mann Whitney U Test, ${ }^{b}$ Kruskall Wallis Test MPV: Mean platelet volume 
Table III. Evaluation of descriptive characteristics according to type of alopecia areata.

\begin{tabular}{|c|c|c|c|c|c|}
\hline & & \multicolumn{3}{|c|}{ Type of Alopecia areata } & \multirow[b]{2}{*}{$\mathrm{p}$} \\
\hline & & Reticular $(\mathrm{n}=31)$ & Ophiasis $(\mathrm{n}=17)$ & Siasphio $(n=23)$ & \\
\hline \multirow{2}{*}{ Age } & Min-Max (Median) & $4-18(13)$ & $1-18(14)$ & 4-17 (13) & \multirow{2}{*}{ b 0.716} \\
\hline & Mean \pm SD & $12.48 \pm 4.90$ & $11.41 \pm 5.46$ & $12.52 \pm 3.87$ & \\
\hline \multirow{2}{*}{ Gender; n (\%) } & Male & $17(54.8)$ & $5(29.4)$ & $10(45.3)$ & \multirow{2}{*}{ d 0.253} \\
\hline & Female & $14(45.2)$ & $12(70.6)$ & $13(56.5)$ & \\
\hline \multirow{2}{*}{ Nail involvement; n (\%) } & No & $28(90.3)$ & $11(64.7)$ & $16(69.6)$ & \multirow{2}{*}{${ }^{\circ} 0.060$} \\
\hline & Yes & $3(9.7)$ & $6(35.3)$ & $7(30.4)$ & \\
\hline \multirow{2}{*}{ Family history; n (\%) } & No & $27(87.1)$ & $17(100)$ & $21(91.3)$ & \multirow{2}{*}{${ }^{\circ} 0.479$} \\
\hline & Yes & $4(12.9)$ & $0(0)$ & $2(8.7)$ & \\
\hline \multirow{2}{*}{ Autoimmune disease; n (\%) } & No & $23(74.2)$ & $16(94.1)$ & $19(82.6)$ & \multirow{2}{*}{${ }^{\circ} 0.260$} \\
\hline & Yes & $8(25.8)$ & $1(5.9)$ & $4(17.4)$ & \\
\hline
\end{tabular}

${ }^{\mathrm{b}}$ Kruskall Wallis Test, ${ }^{\mathrm{C}}$ Fisher Freeman Halton Test, ${ }^{\mathrm{d}}$ Pearson Chi-Square Test

Table IV. Evaluation of descriptive characteristics according to severity of alopecia areta.

\begin{tabular}{|c|c|c|c|c|}
\hline & & \multicolumn{2}{|c|}{ Severity of Alopecia areata } & \multirow{2}{*}{$\mathrm{p}$} \\
\hline & & $\mathrm{S} 1(\mathrm{n}=29)$ & $\mathrm{S} 2(\mathrm{n}=42)$ & \\
\hline \multirow{2}{*}{ Age } & Min-Max (Median) & $1-18(13)$ & $4-18(15)$ & \multirow{2}{*}{${ }^{\mathrm{a}} 0.167$} \\
\hline & Mean \pm SD & $11.28 \pm 5.01$ & $12.90 \pm 4.40$ & \\
\hline \multirow{2}{*}{ Gender; n (\%) } & Male & $12(41.4)$ & $20(47.6)$ & \multirow{2}{*}{${ }^{\mathrm{d}} 0.603$} \\
\hline & Female & $17(58.6)$ & $22(52.4)$ & \\
\hline \multirow{2}{*}{ Nail involvement; n (\%) } & No & $22(75.9)$ & $33(78.6)$ & \multirow{2}{*}{${ }^{\mathrm{d}} 0.788$} \\
\hline & Yes & $7(24.1)$ & $9(21.4)$ & \\
\hline \multirow{2}{*}{ Family history; n (\%) } & No & $28(96.6)$ & $37(88.1)$ & \multirow{2}{*}{ e 0.390} \\
\hline & Yes & $1(3.4)$ & $5(11.9)$ & \\
\hline \multirow{2}{*}{ Autoimmune disease; n (\%) } & No & $25(86.2)$ & $33(78.6)$ & \multirow{2}{*}{${ }^{\mathrm{d}} 0.414$} \\
\hline & Yes & $4(13.8)$ & $9(21.4)$ & \\
\hline
\end{tabular}

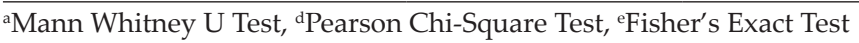

have been emphasized, the etiopathogenesis of AA has not been fully elucidated. ${ }^{9}$ AA is seen especially in young patients and also approximately $60 \%$ of patients have had their first attack before the age of $20 .^{2}$ Although AA can be diagnosed easily by clinical examination, diffuse AA may be diagnostic challenging. In this situation, the dermoscopic findings (broken hair, black dot, yellow dot, exclamation hair) facilitate the differential diagnosis; however, histopathological diagnosis may be needed in a small number of cases. The characteristic histopathologic finding of AA is an intense peribulbar and intrabulbar lymphocytic infiltrate of lymphocytes around the anagen follicles in the appearance of a honey bee colony. ${ }^{9}$

In addition to homeostatic functions, thrombocytes interact with endothelial cells, leukocytes (monocytes, neutrophils, dendritic cells, T-cells), and progenitor cells and allow inflammatory cells to migrate to lesion sites and release abundant quantities of inflammatory cytokine and thus provide an inflammatory environment in the lesion area. It has been reported in recent years that MPV also reflects platelet function and activation and may be a marker of inflammation in different chronic diseases. ${ }^{8}$ 
In our study, the mean MPV levels of the patients with AA were significantly higher than those of the control group. The current hypotheses on the development of AA focuses on the deterioration of autoantigen presentation in the hair follicle as a result of immune privilege in the hair follicles and activation of autoreactive $\mathrm{T}$ lymphocytes. ${ }^{10}$ The autoreactive T-cell response, which is thought to be involved in the pathogenesis of AA, can be genetically determined. ${ }^{10}$ It can also be increased by IL-6 and other cytokines. ${ }^{11}$ Although IL-6 is a proinflammatory cytokine produced by various cells such as fibroblasts, macrophages, $\mathrm{B}$ and $\mathrm{T}$ cells, it plays an important role in the hematopoiesis, immune cell activation, regulation of inflammation, and pathogenesis of autoimmune diseases. ${ }^{12,13}$ Inflammation is known to be an important stimulus for platelets. However, it has been suggested that increased levels of IL- 6 may stimulate platelet production and release large platelets from bone marrow. ${ }^{13}$ Therefore, in our study, the mean MPV levels, which were significantly higher in the patients with AA than in the control group, may be thought to be associated with an increase in young platelets in circulation. When the literature is examined, it is seen that MPV levels vary depending on the severity of systemic inflammation in highand low-grade inflammatory diseases, but the results are contradictory. ${ }^{14}$

When the relationship between the mean MPV level and the age of disease onset, disease duration, disease severity, family history, nail involvement, and accompanying autoimmune disease was evaluated, there was no difference between the AA and control groups. While the MPV measurements showed no statistically significant difference according to the severity of AA, it was remarkable that the MPV measurements were high in the patients with AA severity S2. When the mean MPV level was examined according to the type of AA, it was seen that the mean MPV level was a little bit higher in the reticular pattern, which is more common in the population.
In this study, although there was no significant relationship between the mean MPV level and the clinical activity markers of the patient group (early onset, family history, ophiasis pattern, nail involvement), we think that MPV can be used as a useful marker in the evaluation of AA.

Consequently, AA is a severe and chronic disease in the pediatric age group. Families often refer to a doctor for medical support and treatment because the disease has social and psychological effects. MPV is a simple marker that does not require advanced or expensive technology and also MPV level is determined in each complete blood count. MPV is helpful in assessing clinical activity at a glance in patients with AA. However, prospective studies involving more patients are needed to support our findings.

\section{Ethical approval}

Ethical board approval was received from the Istanbul Anatolia-North Region Public Hospitals Trust (no:10, in 19.10.2015).

\section{Author contribution}

The authors confirm contribution to the paper as follows: study conception and design: NDA; data collection: NDA; analysis and interpretation of results: NDA; draft manuscript preparation: NDA.

\section{Source of funding}

The authors declare the study received no funding.

\section{Conflict of interest}

The authors declare that there is no conflict of interest. 


\section{REFERENCES}

1. Oğuz O. Alopesi areata. Türkderm 2014; 48: 40-44. https://doi.org/10.4274/turkderm.48.s9

2. Dogan S, Evans-Ersoy S, Gonc N, Sahin S. Çocukluk çağında alopesi areata: 89 hastalık bir çalışma. Türkderm 2014; 48: 135-139. https://doi.org/10.4274/ turkderm.69320

3. Gilhar A, Etzioni A, Paus R. Alopecia areata. N Eng J Med 2012; 366: 1515-1525. https://doi.org/10.1056/ NEJMra1103442

4. Alkhalihaf A. Alopecia areata update. Dermatol Clin 2013; 31: 93-108. https://doi.org/10.1016/j. det.2012.08.010

5. Balevi S. Alopesi areata. Türkiye Klinikleri J Int Med Sci 2006; 2: 15-18.

6. Chu SY, Chen YJ, Tseng WC, et al. Comorbidity profiles among patients with alopecia areata: the importance of onset age, a nationwide, populationbased study. J Am Acad Dermatol 2011; 65: 949-956. https://doi.org/10.1016/j.jaad.2010.08.032

7. Sandhaus LM, Meyer P. How useful are CBC and reticulocyte reports to clinicans? Am J Clin Pathol 2002; 118: 787-793. https://doi.org/10.1309/CQGGHYOU-LRKL-GLMP

8. Unal M, Kücük A, Unal Urun G, et al. Mean platelet volume, neutrophil to lyphocyte ratio and platelet to lymphocyte ratio in psoriasis. Türkderm 2015; 49: 112-116. https://doi.org/10.4274/turkderm.57984
9. Hordinsky M, Junqueira AL. Alopecia areata update. Semin Cutan Med Surg 2015; 34: 72-75. https://doi. org/10.12788/j.sder.2015.0160

10. Wang E, McElwee KJ. Etiopathogenesis of alopecia areata: why do our patients get it?. Dermatol Ther 2011; 24: 337-347. https://doi.org/10.1111/j.15298019.2011.01416.x

11. Hirano T. Interleukin 6 and its receptor: ten years later. Int Rev Immunol 1998; 16: 249-284. https://doi. org/10.3109/08830189809042997

12. Cussigh A, Falleti E, Fabris C, et al. Interleukin 6 promoter polymorphisms influence the outcome of chronic hepatitis C. Immunogenetics 2011; 63: 33-41. https://doi.org/10.1007/s00251-010-0491-7

13. Pekiner FN, Aytugar E, Demirel GY, Borahan MO. Interleukin-2, interleukin- 6 and T regulatory cells in peripheral blood of patients with Behçet's disease and recurrent aphthous ulcerations. J Oral Pathol Med 2012; 41: 73-79. https://doi.org/10.1111/j.16000714.2011.01061.x

14. Gasparyan AY, Ayvazyan L, Mikhailidis DP, Kitas GD. Mean platelet volume: a link between thrombosis and inflammation? Curr Pharm Des 2011; 17: 47-58. https://doi.org/10.2174/138161211795049804 